\title{
Interactive effects of disturbance and dispersal directionality on species richness and composition in metacommunities
}

\author{
Florian Altermatt, ${ }^{1,3}$ Sebastian Schreiber, ${ }^{2}$ and Marcel Holyoak ${ }^{1}$ \\ ${ }^{1}$ Department of Environmental Science and Policy, University of California, 1 Shields Avenue, Davis, California 95616 USA \\ ${ }^{2}$ Department of Evolution and Ecology, University of California, 1 Shields Avenue, Davis, California 95616 USA
}

\begin{abstract}
Dispersal among ecological communities is usually assumed to be random in direction, or to vary in distance or frequency among species. However, a variety of natural systems and types of organisms may experience dispersal that is biased by directional currents or by gravity on hillslopes. We developed a general model for competing species in metacommunities to evaluate the role of directionally biased dispersal on species diversity, abundance, and traits. In parallel, we tested the role of directionally biased dispersal on communities in a microcosm experiment with protists and rotifers. Both the model and experiment independently demonstrated that diversity in local communities was reduced by directionally biased dispersal, especially dispersal that was biased away from disturbed patches. Abundance of species (and composition) in local communities was a product of disturbance intensity but not dispersal directionality. High disturbance selected for species with high intrinsic growth rates and low competitive abilities. Overall, our conclusions about the key role of dispersal directionality in (meta)communities seem robust and general, since they were supported both by the model, which was set in a general framework and not parameterized to fit to a specific system, and by a specific experimental test with microcosms.
\end{abstract}

Key words: asymmetric dispersal; density; directionally biased dispersal; ecological gradients; metacommunities; microcosms; species traits.

\section{INTRODUCTION}

Natural landscapes frequently contain variation in habitat patch quality and extent of disturbance among habitat areas. Individually the impact of these factors on species diversity has been extensively explored. This is exemplified by studies of fixed spatial habitat heterogeneity (e.g., Davies et al. 2009), the metacommunity concepts of species sorting and mass effects (e.g., Kneitel and Chase 2004, Leibold et al. 2004, Urban 2004), and the intermediate disturbance hypothesis (IDH; Connell 1978, Shea et al. 2004, Cadotte 2007). Primarily such studies only consider the simplest form of dispersal among local communities. For instance, metacommunity models are usually either based on the spatially implicit Levins (1970) metapopulation model with equal dispersal among patches (Mouquet and Loreau 2002, Amarasekare et al. 2004), or dispersal is inversely related to inter-patch distance, without considering directionality (reviewed in Clobert et al. 2001, Hanski and Gaggiotti 2004). However, the ecology of dispersal is often much more complex than these simple model

Manuscript received 30 May 2010; revised 20 September 2010; accepted 30 September 2010. Corresponding Editor: A. M. de Roos.

${ }^{3}$ Present address: Department of Aquatic Ecology, Eawag: Swiss Federal Institute of Aquatic Science and Technology, Überlandstrasse 133, CH-8600 Dübendorf, Switzerland. E-mail: faltermatt@bluewin.ch representations (e.g., Levin et al. 2003, Nathan et al. 2008).

In many natural systems, landscape structure and physical flows may dictate dispersal of organisms and influence where and how far they disperse (Haddad 1999). Here, we consider the effects of disturbance on species richness when dispersal is directionally biased. Directionally biased dispersal means that most dispersers move predominantly in one spatial direction, and this type of dispersal is likely to be prevalent for species living along environmental gradients or subject to directionally biased environmental currents. There are both theoretical and empirical reasons for considering such forms of dispersal. Existing theoretical work shows that directionally biased dispersal can alter population viability and species coexistence (Vuilleumier and Possingham 2006, Salomon et al. 2010), and that it can be detrimental to metapopulation viability (Elkin and Possingham 2008). Certain types of systems, such as streams, oceanic current systems, or systems with relatively fixed prevailing wind direction, may predispose some organisms to a directional bias in dispersal. Surprisingly there have been few attempts to look at the effects of directionally biased dispersal on diversity in ecological communities, either empirically (but see for example MacIsaac 1996) or theoretically (Salomon et al. 2010).

Another perspective on directionally biased dispersal is to consider the explicit spatial layout of a landscape. 
Often, disturbances do not occur randomly, but there is a spatial pattern defined by landscape properties, and the likelihood of disturbances is spatially correlated across the landscape (Fagan 2002, Peterson 2002). Landscapes may display gradients of disturbance, such as is commonly found along streams, slopes on a mountain, or wave-exposed coastlines (Sousa 1984, MacIsaac 1996). In landscapes with a disturbance gradient, entire clusters of habitat patches may experience similar disturbance regimes, which may influence post-disturbance recovery of local communities. When communities are isolated, recovery from disturbances must happen from within the community from individuals that survived the disturbance. Within a metacommunity, however, local communities also recover from catastrophic disturbances through immigration from neighboring regions. This occurs either via rescue effects, if populations are merely reduced by disturbance, or through colonization if populations have gone extinct. Consequently, the speed of recovery from disturbances may differ between local communities, depending on the spatial arrangement of communities within the landscape (Starzomski and Srivastava 2007). Spatially correlated disturbances may be the norm rather than the exception, and are thought to be especially detrimental not only for local communities, but also for metapopulation persistence (Johst and Drechsler 2003, Elkin and Possingham 2008).

Empirical studies indicate that disturbance and dispersal may be positively or negatively correlated in many natural systems (MacIsaac 1996, Bates et al. 2006, Altermatt et al. 2008). The interaction of dispersal and disturbance gradients might be especially prevalent in species where dispersal is passive, and happens by the same process as disturbance (e.g., wind, water flow), or for species that actively seek or avoid disturbed patches. Empirical case studies, however, usually do not allow a comparison between different disturbances and dispersal gradients and a general approach is necessary to disentangle the effects of spatially correlated disturbance and dispersal on diversity.

Theoretical studies focusing on the effects of aggregated disturbances generally conclude that spatial autocorrelation of disturbances has a negative effect on both abundance and species richness (Fahrig and Merriam 1985, Peterson 2002, Vuilleumier and Possingham 2006). Nonrandom dispersal in these autocorrelated environments can enhance recolonization after disturbance, and hence enhance diversity, or may have negative effects if dispersal is mainly away from areas that have been heavily impacted by disturbance. Only in recent studies has this interaction between disturbance and dispersal been investigated. Using a stochastic, spatially explicit model, Elkin and Possingham (2008) studied the role of directionally biased dispersal in landscapes consisting of less disturbed refuges and more disturbed non-refuge areas. They found that the persistence of metapopulations was reduced when individuals disproportionately dispersed away from refuges and was highest when dispersal was biased toward refuges. Salomon et al. (2010) showed that biased dispersal, which differs between species, is capable of allowing competing species to coexist when they could not do so with random dispersal. Furthermore, some theoretical studies on source-sink dynamics (e.g., Namba and Hashimoto 2004, Armsworth and Roughgarden 2005) and neutral models (e.g., Muneepeerakul et al. 2007) considered directionally biased dispersal. However, we know of no comparable empirical data on metapopulation persistence of one or more species living in landscapes with a disturbance gradients and directionally biased dispersal, and there are no empirical data or theoretical predictions for nonneutral metacommunities.

We developed a general simulation model to test the effects of directionally biased dispersal and disturbance intensity on local and regional species richness. The model covers a wide range of parameter values and was intentionally not parameterized to fit a specific natural system. However, a large number of models have been developed in community ecology in recent years, while empirical confirmation has lagged behind (Agrawal et al. 2007). We thus complemented this model using an independent microcosm experiment with microorganisms (protists, a rotifer, and bacteria). With this twofold approach we can not only explore a wide range of parameters in the model, but also explicitly and independently corroborate our theoretical findings with experimental data. In return, we may evaluate if the empirical results can be generalized by using a general model.

We hypothesized that species diversity and abundance are affected by both dispersal directionality and distance within a metacommunity occurring in a landscape containing a disturbance gradient. We tested four different scenarios by manipulating both dispersal directionality and distance (global dispersal, local dispersal, unidirectional upward dispersal, and unidirectional downward dispersal; Fig. 1) in metacommunities where patches are linearly arranged along a disturbance gradient, and compared these treatments with a no-dispersal control. Compared to other work (e.g., Elkin and Possingham 2008), we not only measured local persistence but also local abundance and density, since local density by itself can interact with dispersal (Clobert et al. 2001, Bates et al. 2006).

\section{Material and Methods}

\section{Simulation model}

Using Lotka-Volterra style competition models, we modeled the dynamics of $m$ competing species living in a stochastically disturbed environment consisting of $n$ patches. For this model, $N_{i k}$ denotes the abundance of species $i$ in patch $k$. The intrinsic rates of growth $r_{i}$ and the competition coefficients $a_{i j}$ for the species are species specific but spatially homogeneous. Individuals in patch 
$k$ emigrate and disperse at a rate $e$ of which a fraction $d_{k l}$ move to patch $l$. Under these assumptions, the competitive dynamics between disturbance events are

$$
\frac{d N_{i k}}{d t}=r_{i} N_{i k}\left(1-\sum_{j=1}^{m} a_{i j} N_{j k}\right)-e N_{i k}+e \sum_{l=1}^{n} d_{l k} N_{i l} .
$$

The dynamics of Eq. 1 are perturbed by stochastic disturbances that are exponentially distributed in time with a mean time $T$ between disturbances. After a disturbance, a fraction $f_{k}$ of all individuals of all species are removed from patch $k$. Between disturbance events, extinction due to demographic stochasticity was mimicked by allowing a species to go locally extinct whenever its abundance within a patch fell below a predetermined threshold ( 0.0001 in our simulations).

In the numerical simulations, there were $m=25$ species and $n=4$ patches. To uncover general patterns of diversity, abundance, and species traits, we intentionally chose species' parameters over a wide range and did not align them to a specific system. The intrinsic rates of growth and the interspecific competition coefficients of the species (i.e., $r_{i}$ and $a_{i j}$ with $i \neq j$ ) were randomly assigned from uniform distributions on the intervals $[0.1,1]$ and $[0,1]$, respectively. Contrary to the randomly assigned interspecific interactions, intraspecific competition coefficients were always set to $a_{i i}=1.0$. We repeated the analysis with the interspecific competition coefficient being randomly assigned from a uniform distribution on the interval $[0,2]$, and thus on average being the same as the intraspecific competition coefficient. As these results are qualitatively similar, we present the latter results only in the Appendix. One hundred communities were created using this distribution of parameter values and all of these communities were used for all combinations of the other model parameters. We also confirmed that the results of our model do not depend on the number of patches or the parameter ranges selected by repeating the analysis for $n=8$ and an alternative interval $[0,1.2]$ of competition coefficients (data not shown).

For each of the dispersal treatments described below, we ran two different rounds of simulations. In the first round, all 25 species were used irrespective of whether they coexist or not in the absence of disturbances (unconstrained species set). However, from previous work (Haddad et al. 2008) we knew that all protozoa species could coexist in undisturbed microcosms. Consequently, we repeated the simulations only using species that could coexist in undisturbed communities (constrained species set). To determine which species could coexist within an undisturbed patch, we simulated the community dynamics in the undisturbed patch for 2000 time units with all species initially at their carrying capacities. The species persisting at the end of the simulation were used for the second round of spatial simulations. This second round of simulations was the only concession of the model to the experiment.

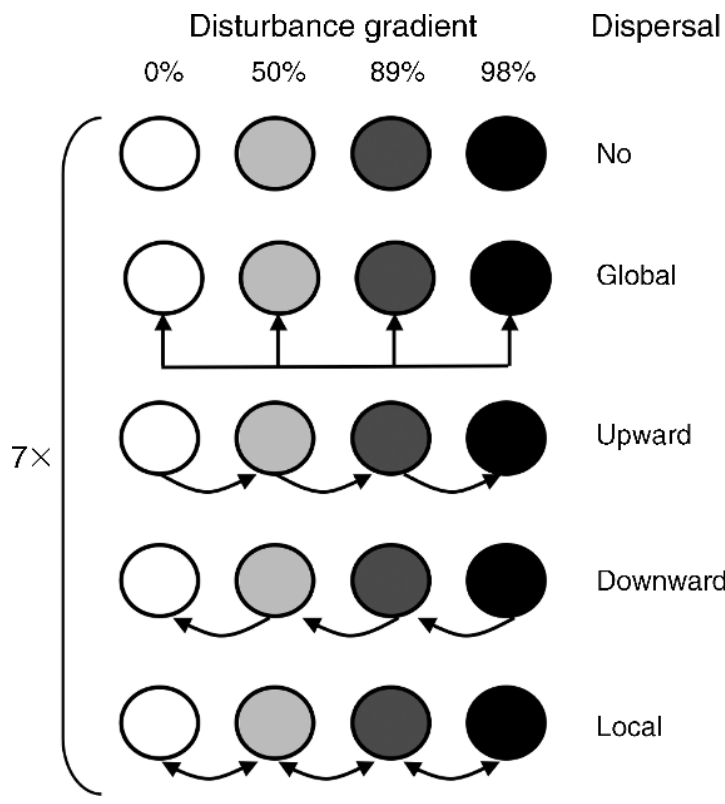

FIG. 1. Setup of the microcosm experiment. In the microcosm experiment, four horizontally aligned patches represent a linear metacommunity with four individual communities. Within each metacommunity, we applied a gradient in disturbance intensity, and communities repeatedly experienced $0 \%, 50 \%, 89 \%$, or $98 \%$ density reduction. Five different dispersal treatments were applied, all replicated seven times. Dispersal directionality was manipulated and is schematically depicted with arrows (arrow tips represent immigration).

Patches were assumed to lie along a one-dimensional disturbance gradient where the fraction of individuals lost to a disturbance in patch $k$ ranged from $f_{1}=0$ to $f_{4}=$ 0.9 in equally spaced increments. The mean time between disturbances was 10 time units. Four forms of dispersal along this gradient were explored (Fig. 1): global random, local random, unidirectional upward along the disturbance gradient (dispersal biased from less disturbed towards more disturbed patches), and unidirectional downward along the disturbance gradient (dispersal biased from more-disturbed patches towards less-disturbed patches). For global random dispersal, equal fractions of emigrating individuals moved to all patches (i.e., $d_{k, l}=1 / 4$ for $k \neq l$ ), corresponding to a Levins-type situation (Levins 1970), where dispersal to all patches is equally likely, and distance between patches does not influence dispersal success. For local random dispersal, half of the emigrating individuals move to the neighboring patch up the disturbance gradient and the other half move to the neighboring patch down the disturbance gradient, whenever possible (i.e., $d_{k, k+1}=1 / 2$ for $k=1, \ldots, 3, d_{k, k-1}=1 / 2$ for $k=$ $2, \ldots, 4$, and $d_{k l}=0$ otherwise). For upward dispersal, all emigrating individuals move from less disturbed patches to more disturbed patches whenever possible (i.e., $d_{k, k+1}$ $=1$ for $k=1, \ldots, 3$ and $d_{k l}=0$ otherwise). For downward dispersal, all emigrating individuals move from more 
disturbed patches to less disturbed patches whenever possible (i.e., $d_{k, k-1}=1$ for $k=2, \ldots, 3$ and $d_{k l}=0$ otherwise). In these last two scenarios, not only is dispersal distance non-random and to neighboring patches, but dispersal directionality is also correlated with the disturbance gradient. The four scenarios can be seen as extremes on a continuous scale, and one can easily come up with natural systems, which match one of the four scenarios. For all forms of dispersal, per-capita dispersal rates were varied over six orders of magnitude, from $1 \times 10^{-6}$ to 1 .

Simulations were run using the LSODE package in $\mathrm{R}$ (R Development Core Team 2008), which solves differential equations numerically, until the metacommunity had experienced 200 disturbances (approximately 2000 time units). In the unconstrained simulations, all 25 species were initiated at their carrying capacity in all patches at the beginning of the simulation. In the constrained simulations, the species that coexist without disturbances were initiated at their carrying capacities in all the patches and the remaining species were excluded from the simulations (i.e., their densities remained zero throughout the simulation). Data from these simulations were recorded for the last 100 disturbances to eliminate transients. This recorded data included local and regional species richness, local and regional abundance of competitors, the average intrinsic rate of growth,

$$
\sum_{i=1}^{25} r_{i} N_{i k} / \sum_{i=1}^{25} N_{i k}
$$

within each patch, and the average interaction strengths

$$
\sum_{i \neq j} a_{i j} N_{i k} N_{j k} / \sum_{i \neq j} N_{i k} N_{j k}
$$

within each patch. In addition, two measures of competitive rank were recorded. The first measure, competitive vulnerability,

$$
\sum_{j \neq i} a_{i j}
$$

quantifies the impact of all other competitors on the species $i$. The second measure, competitive impact,

$$
\sum_{j \neq i} a_{j i}
$$

measures the impact of species $i$ on all other competitors.

\section{Microcosm experiments}

We conducted our experiment in aquatic microcosms containing seven protozoan species, one rotifer species and a set of common freshwater bacteria as food-source. The protozoan species were Colpidium sp., Chilomonas sp., Euglena gracilis, Paramecium aurelia, P. bursaria, Euplotes aediculatus, and Spirostomum sp., while the rotifer remained unidentified (cf. Rotifera sp.). Five of the protozoans and the rotifer were originally collected from a single pond (McGrady-Steed et al. 1997), while Chilomonas sp. and Spirostomum sp. are common freshwater species which came from Carolina Biological Supply Company (Burlington, North Carolina, USA). All species are bacterivores. Euplotes aediculatus and Spirostomum sp. are also capable of feeding on smaller protozoans and Euglena gracilis, Euplotes sp., and $P$. bursaria can also photosynthesize. Microcosms similar to those used here have been widely used to experimentally address questions in community and metacommunity biology, with some focus on dispersal and disturbances (Warren 1996, Kneitel and Chase 2004, Cadotte 2007, Haddad et al. 2008, Davies et al. 2009).

Like Haddad et al. (2008), each replicate patch consisted of a $240-\mathrm{mL}$ glass jar filled with $100 \mathrm{~mL}$ of medium and two autoclaved wheat seeds as a carbon source for bacteria. The medium was a standard soilwater solution, prepared by mixing $2.4 \mathrm{~g}$ of sterilized soil, $0.6875 \mathrm{~g}$ of Protozoan Pellet (Carolina Biological Supply), and $0.1 \mathrm{~g}$ of Herptivite multivitamin mixture (Rep-Cal Research Labs Company, Los Gatos, California, USA) in $1.5 \mathrm{~L}$ of spring water and then sterilizing by steam autoclaving. A day before adding protozoa, this solution was inoculated with $1 \mathrm{~mL}$ of a mixed bacterial culture to provide resources for protozoans. The culture consisted of Bacillus cereus, B. subtilis, and Serratia marcescens obtained from Carolina Biological Supply. All jars were covered with aluminum foil lids to reduce evaporation. Sterile technique was used throughout. Each jar was initiated with a community of all eight protist and rotifer species. Initial population numbers per jar were about 100 individuals for all species but Spirostomum, which naturally occurs at lower densities and was initiated with a population of about 30 individuals per jar. All species persisted at these starting densities, as demonstrated by their persistence in the undisturbed isolated controls. All communities were allowed to grow for one week before disturbance treatments started.

We conducted a factorial experiment in which we varied disturbance intensity and dispersal directionality (Fig. 1). We used metacommunities of four patches and disturbance intensities related to the degree of density reduction by replacing bottle contents with sterilized media. Within each metacommunity, we mimicked a disturbance gradient by subjecting patches to $0 \%, 50 \%$, $89 \%$, or $98 \%$ density reduction. These density reductions are based on previous experiments (Haddad et al. 2008). We disturbed microcosms every five days. We applied dispersal treatments on the same days as the disturbance treatments, resulting in total in seven disturbancedispersal events. Disturbance occurred between emigration and immigration, and migrants were thereby not affected by disturbance. We had the same four dispersal treatments as in the numerical simulations and a nodispersal control, each replicated seven times (Fig. 1). In total, we thus had 35 metacommunities, comprising 140 individual communities. Emigration was the same for all 
patches, and consisted of the removal of $2 \mathrm{~mL}$ of medium (i.e., $8 \mathrm{~mL}$ per metacommunity). The medium in each jar was thoroughly mixed before emigration took place, and these $2 \mathrm{~mL}$ from each jar provided the immigration material. This amount of medium was sufficient to contain emigrants due to the small size of the protists and their usually high population densities (Haddad et al. 2008). Emigration also occurred in the no-dispersal control, to control for handling effects or the small reduction in population size. Immigration was applied according to the different dispersal treatments. In the global dispersal treatment, all $8 \mathrm{~mL}$ were mixed before redistribution, and in the local dispersal treatment the $2 \mathrm{~mL}$ were split and half went one way and half the other way. To keep total immigration rates per metacommunity constant across dispersal treatments and to control for emigration at the end of the disturbance gradient, in all treatments $6 \mathrm{~mL}$ of "immigration" occurred within the whole metacommunity, and the missing $2 \mathrm{~mL}$ of volume was replaced with sterilized medium (to control for the lost medium). To prevent population collapse due to nutrient depletion, we replaced $10 \mathrm{~mL}$ of microcosm contents with sterile medium in each undisturbed community after three weeks (Haddad et al. 2008); we did not conduct this procedure in the disturbed patches because medium was already being replaced through the disturbance process.

After 37 days (2 days after the last disturbancedispersal treatment), we estimated the density of the eight study species in each replicate with a stereo microscope (20-40× magnification), thereby also recording presence and absence of each species. We thoroughly mixed the contents of each jar and took a subsample of $10 \mathrm{~mL}$. Because of the different species' sizes and densities, volumes censused were adjusted to obtain an adequate density estimate (for details, see Haddad et al. 2008, Davies et al. 2009).

\section{Statistical analyses}

To test for the effect of disturbance intensity and dispersal directionality on species richness, we used a split-plot ANOVA, where the blocking was the different replicates of the metacommunities, and disturbance was nested within dispersal directionality. This analysis gave us information on the significance of each factor, but did not allow comparison of the five dispersal treatments. Therefore, after confirming the overall significance of dispersal directionality, we conducted ANOVAs, comparing two dispersal treatments at a time. We did four orthogonal a priori comparisons: downward dispersal vs. the no-dispersal control; local dispersal vs. upward dispersal; local dispersal vs. downward dispersal; and local dispersal vs. global dispersal. The first comparison focuses on the effect of dispersal per se, the second and third comparisons focus on dispersal directionality, and the last comparison focuses on dispersal distance rather than directionality. We then analyzed the effect of disturbance intensity and directionality on species density, using a factorial MANOVA. Species' density data were $\log _{10}$-transformed to meet normality assumptions, and only replicates with species present were included in analyses. The resulting unbalanced design did not allow for the blocked structure, and we used a factorial approach. Finally, we focused on whole metapopulations and metacommunities. We analyzed the effect of dispersal directionality on the percentage of occupied patches for each species within a metacommunity with a MANOVA, using arcsine-transformed proportion data. We limited the analysis to species that showed variation in occupancy, and excluded species that occurred in $>95 \%$ of all patches. After confirming the overall significance of the dispersal treatment, we conducted four contrasts of the same type as for species richness. All analyses were conducted using R ( R Development Core Team 2008).

\section{RESUlts}

\section{Simulation model}

In simulations, regional species richness decreased in response to increased dispersal for all dispersal directionalities (Fig. 2A). While the dispersal directionality had little effect on regional species richness at lower and intermediate dispersal rates, upward dispersal at the two highest dispersal rates resulted in $10 \%$ to $60 \%$ lower richness than all other dispersal directionalities. Local species richness was maximized at intermediate dispersal rates (Fig. 2B). At these intermediate dispersal rates, global random dispersal resulted in the highest local species richness while downward dispersal to less disturbed patches resulted in the lowest local species richness. Global random dispersal achieved the highest local richness by spreading all species relatively uniformly across the landscape (Fig. 3B, G). In contrast, while downward dispersal slightly increased local richness in the less disturbed patches, upward dispersal to more disturbed patches resulted in greater increases in local richness in the more disturbed patches (compare Fig. 3D-C). While local random dispersal can produce higher levels of local species richness than upward dispersal, these two dispersal patterns produce similar levels of local species richness at low dispersal rates $(\log [$ dispersal rate $] \leq 4$; Fig. $2 \mathrm{~B}$, Fig. 3C, E). At high dispersal rates, local species richness strongly decreases for both upward and downward dispersal (Fig. 2B). These patterns were qualitatively also very consistent when using an interspecific competition coefficient sampled from $[0,2]$, but at a generally lower species richness (see Appendix: Figs. A1 and A2).

While local diversity typically decreased along the disturbance gradients (Fig. 3A-K), a notable exception occurred for unconstrained communities with upward or local random dispersal (Fig. 3C, E). For these communities, maximal local diversity was achieved in patches with intermediate disturbance frequencies. Aside from this notable exception, the results of simulations with a constrained species set were consistent (see Appendix: 

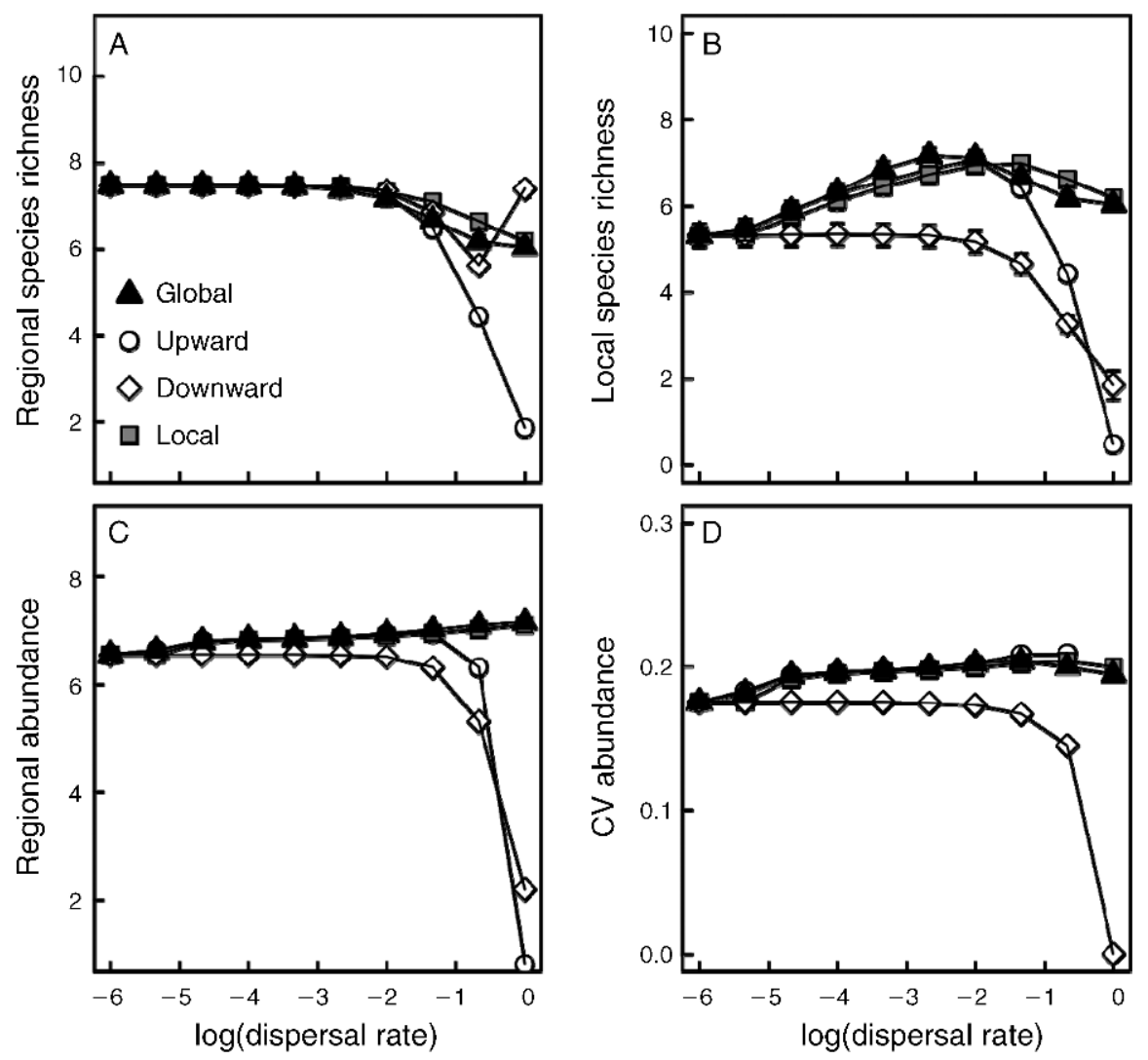

FIG. 2. Simulated (A) regional species richness, (B) local species richness, (C) abundance, and (D) coefficient of variation (CV) of regional abundance (means $\pm \mathrm{SE}$ ) are plotted as a function of the per capita dispersal rate $e$ with different curves corresponding to different dispersal patterns. The simulations started with an unconstrained set of species.

Fig. A3). By definition, constraining the initial species set resulted in lower regional diversity at low rates of dispersal, but local diversity and abundance measures were otherwise very similar.

Regional abundance of all of the competing species increased slightly with increased dispersal rates (Fig. $2 \mathrm{C})$. For sufficiently positive dispersal rates $\left(e>10^{-4}\right)$, downward dispersal produced lower regional abundance than all other dispersal patterns. However, the magnitude of this effect was slight except at high dispersal rates, in which case most individuals moved to the undisturbed patch. Reversed, at high upward dispersal, all organisms eventually end up in the highly disturbed patch. Temporal variability in regional abundance increased slightly with dispersal for all dispersal patterns allowing individuals to move from less disturbed patches to more disturbed patches (Fig. 2D). This increase occurs for random dispersal because less disturbed patches support higher abundances and, therefore, increasing the per capita dispersal rate increased the net dispersal of individuals from less disturbed patches to more disturbed patches.

At intermediate dispersal rates, disturbance but not dispersal directionality had an effect on species traits (see Appendix: Fig. A4). Species persisting in more disturbed patches had higher intrinsic growth rates, were competitively inferior (i.e., had greater competitive vulnerability), and exhibited higher interaction strengths which is likely related to lower species richness in the more disturbed patches. Namely, in the highly disturbed environments, intraspecific interactions are more likely than interspecific interactions and interspecific competition coefficients (mean of $a_{i j}$ with $i \neq j$ is 0.6 ) were, on average, smaller than intraspecific competition coefficients $\left(a_{i i}=1\right)$.

\section{Microcosm experiments}

Regional species richness within microcosm metacommunities was identical across all dispersal treatments, with none of the eight species being lost. In common with the simulations (and almost identical to the simulations with the unconstrained species set; Fig. $3 \mathrm{~F}-\mathrm{K}$ ), local species richness decreased along the disturbance gradient (Fig. 3L-P, Table 1). Also, local species richness in microcosms was significantly influenced by both dispersal directionality and the interaction between disturbance intensity and dispersal directionality (Fig. 3L-P, Table 1). Local species richness was highest in the global random dispersal treatment and lowest in the downward dispersal 
Model, unconstrained

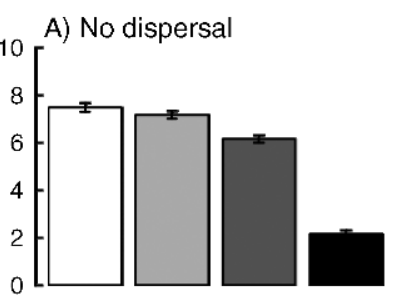

B) Global dispersal

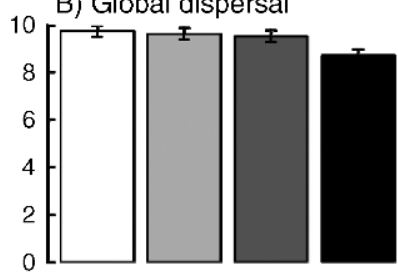

C) Upward dispersal

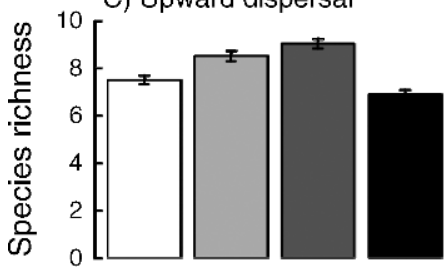

D) Downward dispersal

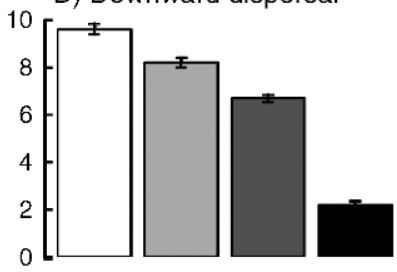

E) Local dispersal

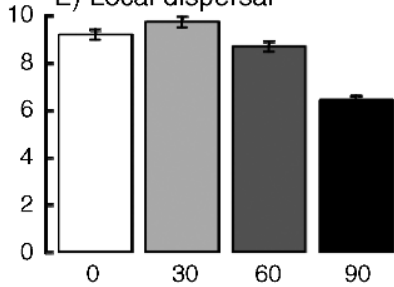

Model, constrained

F) No dispersal

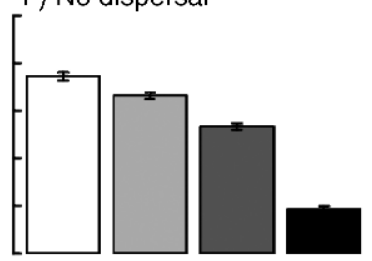

G) Global dispersal

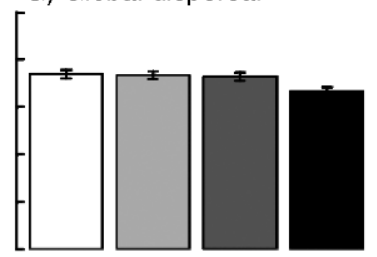

H) Upward dispersal

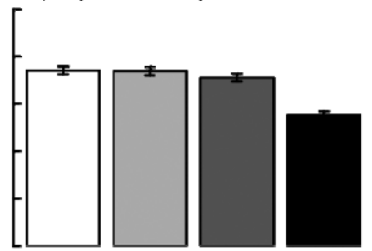

J) Downward dispersal

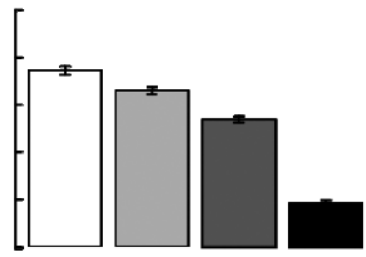

K) Local dispersal

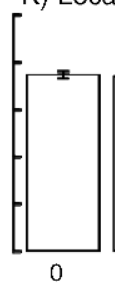

Experiment

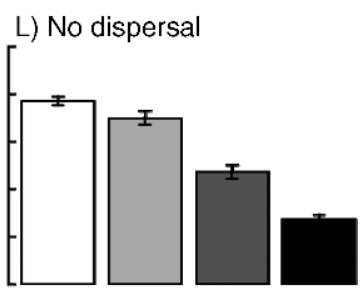

M) Global dispersal

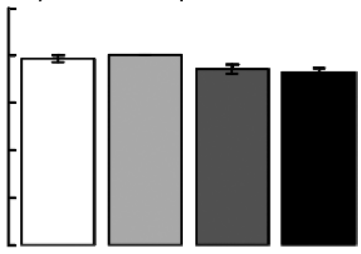

N) Upward dispersal

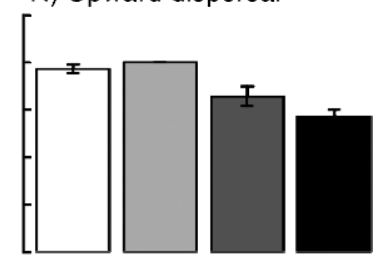

O) Downward dispersal

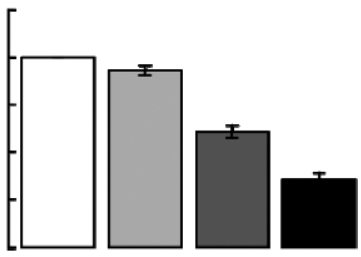

P) Local dispersal

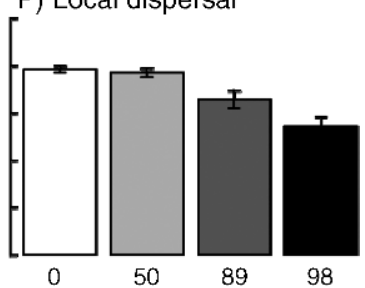

Disturbance intensity (\%)

FIG. 3. Species richness (mean $\pm \mathrm{SE}$ ) within single communities in response to disturbance intensity and different dispersal directionalities in $(\mathrm{A}-\mathrm{K})$ simulation models and $(\mathrm{L}-\mathrm{P})$ microcosm experiments. Panels $\mathrm{A}-\mathrm{E}$ are simulations that started with an unconstrained set of species, while panels $\mathrm{F}-\mathrm{K}$ are simulations in which the species set was constrained to species that could coexist under undisturbed conditions. Disturbance intensity in the simulation models was $0 \%, 30 \%, 60 \%$, and $90 \%$, and the dispersal rate $e$ $\approx 0.002$. Based on previous experiments, the disturbance intensity in microcosm experiments was $0 \%, 50 \%, 89 \%$, and $98 \%$. Note that there is no panel I.

treatment. Species richness in the other two dispersal treatments was intermediate. Comparisons of pairs of dispersal treatments were consistent with the main analysis including all dispersal treatments. In all four comparisons, disturbance intensity had a negative effect on local species richness (Table 1). Local species richness was significantly lower at local random dispersal vs. global random dispersal (Table 1B) and at downward dispersal vs. local dispersal (Table 1D). There was no significant difference in local species richness between local random dispersal vs. upward dispersal and nodispersal vs. downward dispersal (Table 1C, E).

Density of the different species within patches significantly decreased with increasing disturbance intensity $\left(F_{24,123}=5.47, P<0.0001\right)$, but was - contrary to species richness and in accordance to the numerical 
TABLE 1. ANOVA on the effect of dispersal directionality, disturbance intensity, and their interaction on species richness.

\begin{tabular}{|c|c|c|c|c|c|}
\hline Effect & $\mathrm{df}$ & SS & MS & $F$ & $P$ \\
\hline \multicolumn{6}{|c|}{ A) Full model with all levels of dispersal directionality } \\
\hline Block & 6 & 6.89 & 1.15 & & \\
\hline Dispersal & 4 & 87.5 & 21.9 & 44.1 & $<0.0001$ \\
\hline Disturbance & 3 & 215 & 71.5 & 206 & $<0.0001$ \\
\hline Interaction & 12 & 66.2 & 5.52 & 15.9 & $<0.0001$ \\
\hline Error & 90 & 31.2 & 0.35 & & \\
\hline \multicolumn{6}{|c|}{ B) Subset of local dispersal vs. global dispersal } \\
\hline Block & 6 & 3.86 & 0.64 & & \\
\hline Dispersal & 1 & 7.88 & 7.88 & 10.5 & 0.018 \\
\hline Disturbance & 3 & 22.3 & 7.45 & 23.6 & $<0.0001$ \\
\hline Interaction & 3 & 7.05 & 2.35 & 7.45 & 0.0005 \\
\hline Error & 36 & 11.4 & 0.32 & & \\
\hline \multicolumn{6}{|c|}{ C) Subset of local dispersal vs. upward dispersal } \\
\hline Block & 6 & 8.21 & 1.37 & & \\
\hline Dispersal & 1 & 0.16 & 0.16 & 0.36 & 0.57 \\
\hline Disturbance & 3 & 49.9 & 16.6 & 34.5 & $<0.0001$ \\
\hline Interaction & 3 & 0.48 & 0.16 & 0.33 & 0.8 \\
\hline Error & 36 & 17.4 & 0.48 & & \\
\hline \multicolumn{6}{|c|}{ D) Subset of local dispersal vs. downward dispersal } \\
\hline Block & 6 & 5.68 & 0.95 & & \\
\hline Dispersal & 1 & 17.2 & 17.2 & 23.1 & 0.003 \\
\hline Disturbance & 3 & 130 & 43.2 & 120 & $<0.0001$ \\
\hline Interaction & 3 & 16.7 & 5.54 & 15.3 & $<0.0001$ \\
\hline Error & 36 & 13 & 0.36 & & \\
\hline \multicolumn{6}{|c|}{ E) Subset of no dispersal vs. downward dispersal } \\
\hline Block & 6 & 3.93 & 0.65 & & \\
\hline Dispersal & 1 & 0.88 & 0.88 & 1.75 & 0.23 \\
\hline Disturbance & 3 & 228 & 75.9 & 254 & $<0.0001$ \\
\hline Interaction & 3 & 0.20 & 0.07 & 0.22 & 0.88 \\
\hline Error & 36 & 10.8 & 0.3 & & \\
\hline
\end{tabular}

model-not influenced by dispersal directionality $\left(F_{32,168}=0.69, P=0.89\right)$ or its interaction with disturbance intensity $\left(F_{48,264}=1.07, P=0.36\right.$; Fig. 4$)$.

Three of the eight study species (Chilomonas sp., Colpidium sp. and $P$. aurelia) persisted in $>95 \%$ of all local communities. The other five species persisted in $47-$ $88 \%$ of local communities, and occupancy (percentage of patches occupied) differed significantly between dispersal directionality treatments (MANOVA on the effect of dispersal directionality on occupancy: $F_{20,116}=$ 3.1, $P<0.0001$; see Appendix: Fig. A5). Consistent with the results for species richness within patches (Table 1), but only marginally significant, occupancy was lower at local random dispersal vs. global random dispersal (orthogonal contrast, $t=1.9, P=0.068$ ). Also, the occupancy was significantly lower at downward dispersal compared to the local random dispersal (orthogonal contrast, $t=3.3, P=0.0026)$. The occupancy did not differ between local random dispersal and the upward dispersal (orthogonal contrast, $t=1.7, P=0.11$ ), nor between no-dispersal and downward dispersal (orthogonal contrast, $t=1.7, P=0.11)$.

\section{Discussion}

Both the results of our general model and our more specific microcosm experiment showed that dispersal directionality can have strong effects on species diversity and occupancy of metacommunities in a landscape with a disturbance gradient (Figs. 2 and 3 and Table 2). Most strikingly, directionally biased downward dispersal (dispersal from more-disturbed patches towards lessdisturbed patches) produced significantly lower local species diversity and lower patch occupancy within metacommunities compared to equivalent rates of local random dispersal (equal per capita dispersal rates up and down the disturbance gradient). To our knowledge, this is the first theoretical and empirical demonstration that dispersal directionality and disturbance have a combined effect on species richness in metacommunities.

It is generally acknowledged that global dispersal in the sense of Levins (1970) is an oversimplification and may be rarely seen in natural systems. Dispersal is usually inversely related to distance between patches (Clobert et al. 2001), and in many natural systems is directionally biased away from or towards disturbed patches (MacIsaac 1996, Bates et al. 2006). Thus, models using global or unbiased dispersal kernels not only provide an incomplete understanding of metapopulation persistence (see also Elkin and Possingham 2008) but, as shown here (Figs. 2 and 3), also overestimate expected species richness.

In general, our results are consistent with prior work, which separately studied either directionally biased dispersal or spatially autocorrelated disturbances (Ovas- 
A) No dispersal

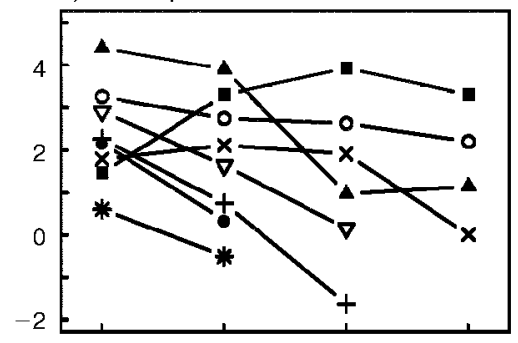

C) Upward dispersal

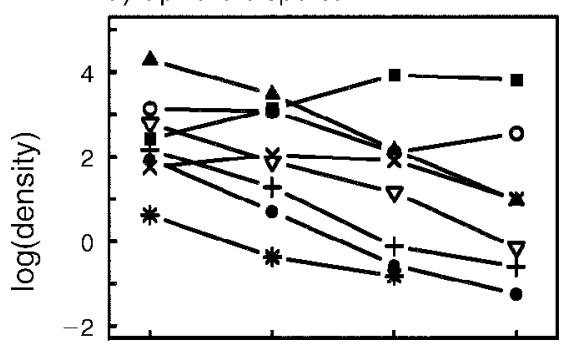

E) Local dispersal

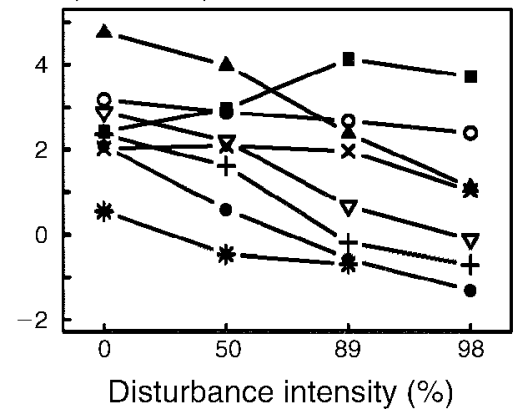

B) Global dispersal

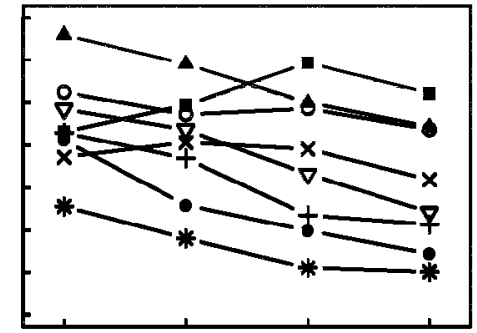

D) Downward dispersal

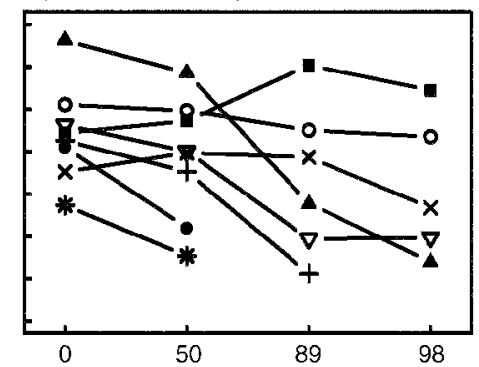

Disturbance intensity (\%)

- Chilomonas sp.

- Colpidium sp.

- Euglena gracilis

+ Euplotes aediculatus

$\times$ Paramecium aurelia

- Paramecium bursaria

$\nabla$ Rotifer

* Spirostomum sp.

FIG. 4. Mean density of each protozoan species within local communities in the microcosm experiment in response to disturbance intensity (disturbance intensity $0 \%, 50 \%, 89 \%$, and $98 \%$ ) and different dispersal directionalities. For clarity, only mean values of density are given (without measures of variability).

kainen and Hanski 2002, Johst and Drechsler 2003, Vuilleumier and Possingham 2006). Elkin and Possingham (2008) were the first to combine these effects, showing that directionally biased dispersal away from undisturbed refuges reduced metapopulation persistence of single species in theoretical simulations. Our theoretical work extends the interaction of disturbance and dispersal directionality from metapopulations to metacommunities, and is complemented with experiments with protozoa and rotifers in microcosms (Fig. 3; Appendix: Fig. A5). Our microcosm experiment was conducted simultaneously with the model, and, to gain generality, the model was intentionally not parameterized to fit the experimental system. Nevertheless, we find surprisingly consistent patterns using those two independent and different approaches (Fig. 3). Since the effect of dispersal directionality on local species richness in the model was seen for dispersal rates spanning several orders of magnitude (Fig. 2B), we think that the results are rather general and not restricted to a specific setup or natural system. Also, the effect was strongest at intermediate - and thus possibly most realistic - dispersal rates.

It is important to note that we intentionally made some simplifications in our model (and partially in the experiment) to accommodate understanding of patterns and processes, which conceptually follows previous work on metacommunities (e.g., Mouquet and Loreau 2003). We assumed that growth rates and competition coefficients, for each species, are spatially homogeneous; that dispersal rate is identical for all species on all patches; and that dispersal directionality is identical across all species on each patch. Finally, the study focuses only on linear landscapes. Thus, our results should be interpreted in that context, and relaxing these assumptions, especially using more complex landscape patterns (e.g., Muneepeerakul et al. 2007), may be worthwhile for future studies.

One of our results provides an interesting contrast to Elkin and Possingham's simulations (2008). They found that directionally biased dispersal from undisturbed refuge patches to disturbed patches reduced metapopu- 
TABLE 2. Overview of the main results and comparison if the results of the model and the experiment are in agreement.

\begin{tabular}{|c|c|c|c|}
\hline Variable of interest & Simulation model & Protozoa experiment & Agree? \\
\hline $\begin{array}{l}\text { Regional diversity relative } \\
\text { to the four dispersal } \\
\text { directionalities }\end{array}$ & $\begin{array}{l}\text { Regional species diversity is mostly not } \\
\text { affected by the four different dispersal } \\
\text { directionalities (except at high } \\
\text { dispersal rates, where regional diversity } \\
\text { is lower in the upward dispersal } \\
\text { treatment compared to the other three } \\
\text { dispersal treatments; Fig. } 2 \mathrm{~A} \text { ). }\end{array}$ & $\begin{array}{l}\text { Regional diversity is not affected by the } \\
\text { four dispersal treatment (Fig. } 3 \mathrm{M}-\mathrm{P} \text { ). }\end{array}$ & (yes) \\
\hline $\begin{array}{l}\text { Regional diversity relative } \\
\text { to different dispersal } \\
\text { rates }\end{array}$ & $\begin{array}{l}\text { Regional diversity decreases with } \\
\text { increasing dispersal rates (Fig. } 2 \mathrm{~A} \text { ). }\end{array}$ & & NA \\
\hline $\begin{array}{l}\text { Local diversity relative to } \\
\text { the four dispersal } \\
\text { directionalities }\end{array}$ & $\begin{array}{l}\text { Local diversity is affect by the dispersal } \\
\text { directionality: at intermediate dispersal } \\
\text { rates, local diversity from highest to } \\
\text { lowest is given by global }>\text { local }> \\
\text { upward }>\text { downward; Figs. } 2 \mathrm{~B} \text { and } 3 \text { ). }\end{array}$ & $\begin{array}{l}\text { Local diversity is affect by the dispersal } \\
\text { directionality (local diversity from } \\
\text { highest to lowest is given by global }> \\
\text { upward }>\text { local }>\text { downward; Fig. } 3 \text { ). }\end{array}$ & yes \\
\hline $\begin{array}{l}\text { Local diversity relative to } \\
\text { disturbance rate (no } \\
\text { dispersal) }\end{array}$ & $\begin{array}{l}\text { Local diversity decreases at higher rates } \\
\text { of disturbance (Fig. 3A, F). }\end{array}$ & $\begin{array}{l}\text { Local diversity decreases at higher rates } \\
\text { of disturbance (Fig. } 3 \mathrm{~L}) \text {. }\end{array}$ & yes \\
\hline $\begin{array}{l}\text { Local diversity relative to } \\
\text { different dispersal rates }\end{array}$ & $\begin{array}{l}\text { Local diversity is highest at intermediate } \\
\text { dispersal rates, but the magnitude of } \\
\text { this effect depends strongly on } \\
\text { dispersal directionality (Fig. 2B). }\end{array}$ & & NA \\
\hline Abundance/density & $\begin{array}{l}\text { Abundance (regional) is not affected } \\
\text { by dispersal directionality (Fig. } 2 \mathrm{C} \text { ). }\end{array}$ & $\begin{array}{l}\text { Abundance (local and regional) is not } \\
\text { affected by dispersal directionality } \\
\text { (Fig. 4). }\end{array}$ & yes \\
\hline $\begin{array}{l}\text { Species traits (intrinsic rate } \\
\text { of growth, competitive } \\
\text { vulnerability, and } \\
\text { interaction strengths) }\end{array}$ & $\begin{array}{l}\text { Species traits are only affected by the } \\
\text { disturbance intensity in local patches, } \\
\text { but not by dispersal directionality } \\
\text { (Appendix: Fig. A4). }\end{array}$ & & NA \\
\hline
\end{tabular}

Notes: The results always refer to metacommunities in a linear landscape with a disturbance gradient. Different dispersal directionalities are applied (see Fig. 1). Since some results are unique to the model, comparisons are not applicable (NA). In cases where the model's results agree with the experiment over most, but not all, parameter space explored in the model, the "yes" was put in parentheses.

lation viability for single species in simulated landscapes. We, however, found that biased dispersal away from the more disturbed patches had the greatest negative effects on local species diversity and patch occupancy (Fig. 2B), but did not impact metacommunity (or metapopulation) persistence. A possible explanation could be that in Elkin and Possingham's model a disturbance event eliminated the whole population within a patch, while in our model and experiment a disturbance caused merely a reduction in local population size. Thus, in Elkin and Possingham's model, all individuals dispersing into patches which are subject to a disturbance will be killed and lost for the metapopulation, ultimately reducing metapopulation persistence. This process can be seen as a "negative" mass effect, and no dispersal can occur away from disturbed patches. In our case, however, we also incorporated within-population dynamics, and disturbances only reduced population density, allowing dispersal from a patch after a disturbance event. Our approach is less restrictive, and may be more realistic for many natural systems (Sousa 1984).

Ecological communities are known to be organized at different spatial scales, and interactions between biotic and abiotic processes at these different scales determine local and regional patterns of species richness (Levin 1992, Mouquet and Loreau 2002, 2003, Ricklefs 2008). Moquet and Loreau $(2002,2003)$ examined models of competitive metacommunities with symmetric, global dispersal and spatial heterogeneity. Under equilibrium conditions, they found that local species richness is maximized at intermediate dispersal rates, and regional species richness decreases with dispersal rates. Our simulations show that these conclusions extend to metacommunities exhibiting directionally biased or random movement along disturbance gradients (Fig. 2B). The maximization of local species richness at intermediate dispersal rates, however, is most pronounced for global random dispersal and weakest for downward dispersal. Hence, directionally biased dispersal may make these patterns difficult to detect in natural systems. These model predictions are partially supported by a recent meta-analysis of 50 experiments (Cadotte 2006) and experiments on metacommunities of algae species (Matthiessen and Hillebrand 2006). The meta-analysis for animals and the experiments found that local diversity is maximized at intermediate dispersal rates. However, no significant trend was found in the meta-analysis of plant species. Cadotte's metaanalysis also found a significant negative effect of dispersal on regional diversity, but no significant effect was found in Matthiessen and Hillebrand's experimental work.

While local diversity was affected both by the disturbance intensity and the dispersal directionality, 
local abundance or density of species was only affected by the disturbance intensity. Dispersal directionality did not affect abundance for most of the dispersal rates in the model (Fig. 2C) and in the experiment (Fig. 4). In other words, dispersal directionality modifies presence and absence of a species, but once present the species bounces back to a constant abundance or density. Local density is then only affected by disturbance intensity. Consistent with the predictions by Loreau et al. (2003), we also found in the model that abundance generally increased with increasing dispersal rates.

For most species, and in accordance with Haddad et al. (2008), local densities in the experimental metacommunities were lower at high disturbance intensities (Fig. 4). In such high-disturbance environments, only species with a high intrinsic rate of growth may persist (Haddad et al. 2008). Consistently, the model only predicted differences in species traits within local patches due to disturbance intensity (see Appendix: Fig. A4), but not due to dispersal directionality: on average, the intrinsic rate of growth is much higher for species found in highly disturbed patches than in less disturbed patches and species with higher competitive rank were more common in less disturbed patches and less common in highly disturbed patches.

The results of the model help us to generalize from our specific lab experimental system, since the formalism of the model shares common features with many other competitive metacommunity models (e.g., Mouquet and Loreau 2003, Büchi et al. 2009, Salomon et al. 2010). As mentioned, the results of the model (at intermediate dispersal rates) and the experiment are consistent, even though they were not parameterized to each other (Fig. $3)$. The results of the model with an unconstrained species set (Fig. 3A-E) differ only in two aspects from the results of the experiments (Fig. 3L-P): In the unconstrained model, regional diversity was affected by dispersal directionality at medium to high dispersal rates (Fig. 2A), while this was not the case in the experiment. Also, upward and local dispersal in the unconstrained model let local diversity peak in patches with intermediate disturbance rates (Fig. 3C, E), while in the experiment local diversity decreased with increasing disturbance rates (Fig. 3N, P). This difference, however, vanished when a constrained species set was used in the model, and local diversity in the model and the experiment became basically identical (Fig. 3F-P). In the simulations with a constrained species a humped shaped pattern in local diversity at intermediate disturbance rates is not possible, as the maximal local diversity occurs in the undisturbed patches. Since all protozoa and rotifer species are known for their ability to coexist in undisturbed patches (Haddad et al. 2008), regional diversity is per se expected to be the same for all experimental metacommunities. Local diversity as well as occupancy for individual protozoa and rotifer species was then only reduced at higher disturbance intensities. Species richness was lowest in the isolated patches of the no-dispersal control, where only disturbance tolerant species could persist. In the four dispersal treatments, species richness in disturbed patches increased, depending on the dispersal directionality and dispersal distance. The precise quantitative similarity between the local species diversity in the constrained simulations (Fig. 3F$\mathrm{K})$ compared to the experimental treatments (Fig. 3L-P) is likely fortuitous, i.e., an arbitrary result of our choice of parameters. However the general patterns of diversity across treatments in simulations was highly consistent regardless of our choice of parameter values. In the model started with an unconstrained species set, however, species richness was also reduced in isolated, undisturbed patches because of competitive exclusion. Since the effect of dispersal directionality on regional species richness was strongest in the model at high rates of dispersal, it is possible that at low rates of dispersal, competitive interactions reduce regional species richness initially, but allow coexistence independent of dispersal directionality (Fig. 2A).

A recent model suggests that environmental spatial structures and disturbances affect life-history traits of species in metacommunities (Büchi et al. 2009). Among others, dispersal ability of species increased in disturbed systems, and affected species richness (Büchi et al. 2009). With our model and experiment we have shown that for a given disturbance gradient in a landscape, species richness may be affected by dispersal directionality alone, and thus not necessarily cause or depend on differences in species traits. In the model, the traits of the persisting, and thus selected, species did not differ between the dispersal treatments (see Appendix: Fig. A4). This suggests that directionally biased dispersal does not favor species with certain traits, which then influence the local diversity, but that directionally biased dispersal per se reduces species richness, independently of their traits. Taken together, we conclude that directionally biased dispersal and disturbance gradients, both of which may be common in natural landscapes, are powerful actors in interactively shaping local and regional species diversity.

\section{ACKNOWLEDGMents}

We thank A. Bieger for help during the experiment. P. Amarasekare made helpful comments on the manuscript. F. Altermatt was supported by the Swiss National Science Foundation (PBBSP3-124435). S. Schreiber was supported by NSF DMS-0517987 and OCE-0623224. M. Holyoak was supported by NSF-DEB 0414465.

\section{Literature Cited}

Agrawal, A. A., et al. 2007. Filling key gaps in population and community ecology. Frontiers in Ecology and the Environment 5:145-152.

Altermatt, F., V. I. Pajunen, and D. Ebert. 2008. Climate change affects colonisation dynamics in a metacommunity of three Daphnia species. Global Change Biology 14:1209-1220.

Amarasekare, P., M. F. Hoopes, N. Mouquet, and M. Holyoak. 2004. Mechanisms of coexistence in competitive metacommunities. American Naturalist 164:310-326. 
Armsworth, P. R., and J. E. Roughgarden. 2005. The impact of directed versus random movement on population dynamics and biodiversity patterns. American Naturalist 165:449-465.

Bates, A. J., J. P. Sadler, and A. P. Fowles. 2006. Conditiondependent dispersal of a patchily distributed riparian ground beetle in response to disturbance. Oecologia 150:50-60.

Büchi, L., P.-A. Christin, and A. H. Hirzel. 2009. The influence of environmental spatial structure on the life-history traits and diversity of species in a metacommunity. Ecological Modelling 220:2857-2864.

Cadotte, M. W. 2006. Dispersal and species diversity: a metaanalysis. American Naturalist 167:913-924.

Cadotte, M. W. 2007. Competition-colonization trade-offs and disturbance effects at multiple scales. Ecology 88:823-829.

Clobert, J., E. Danchin, A. A. Dhondt, and J. D. Nichols, editors. 2001. Dispersal. Oxford University Press, Oxford, UK.

Connell, J. H. 1978. Diversity in tropical rainforests and coral reefs. Science 199:1302-1310.

Davies, K. F., M. Holyoak, K. A. Preston, V. A. Offeman, and Q. Lum. 2009. Factors controlling community structure in heterogeneous metacommunities. Journal of Animal Ecology 78:937-944.

Elkin, C. M., and H. Possingham. 2008. The role of landscapedependent disturbance and dispersal in metapopulation persistence. American Naturalist 172:563-575.

Fagan, W. F. 2002. Connectivity, fragmentation, and extinction risk in dendritic metapopulations. Ecology 83:3243-3249.

Fahrig, L., and G. Merriam. 1985. Habitat patch connectivity and population survival. Ecology 66:1762-1768.

Haddad, N. M. 1999. Corridor and distance effects on interpatch movements: a landscape experiment with butterflies. Ecological Applications 9:612-622.

Haddad, N. M., M. Holyoak, T. M. Mata, K. F. Davies, B. A. Melbourne, and K. Preston. 2008. Species' traits predict the effects of disturbance and productivity on diversity. Ecology Letters 11:348-356.

Hanski, I., and O. E. Gaggiotti, editors. 2004. Ecology, genetics and evolution of metapopulations. Elsevier Academic Press, Amsterdam, The Netherlands.

Johst, K., and M. Drechsler. 2003. Are spatially correlated or uncorrelated disturbance regimes better for the survival of species? Oikos 103:449-456.

Kneitel, J. M., and J. M. Chase. 2004. Disturbance, predator, and resource interactions alter container community composition. Ecology 85:2088-2093.

Leibold, M. A., M. Holyoak, N. Mouquet, P. Amarasekare, J. M. Chase, M. F. Hoopes, R. D. Holt, J. B. Shurin, R. Law, D. Tilman, M. Loreau, and A. Gonzalez. 2004. The metacommunity concept: a framework for multi-scale community ecology. Ecology Letters 7:601-613.

Levin, S. A. 1992. The problem of pattern and scale in ecology. Ecology 73:1943-1967.

Levin, S. A., H. Muller-Landau, R. Nathan, and J. Chave. 2003. The ecology and evolution of seed dispersal. Annual Review of Ecology and Systematics 34:575-604.

Levins, R. 1970. Extinction. Pages 77-107 in E. Gerstenhaber, editor. Some mathematical problems in biology. American Mathematical Society, Providence, Rhode Island, USA.

Loreau, M., N. Mouquet, and A. Gonzalez. 2003. Biodiversity as spatial insurance in heterogeneous landscapes. Proceedings of the National Academy of Sciences USA 100:12765-12770.
MacIsaac, H. J. 1996. Population structure of an introduced species (Dreissena polymorpha) along a wave-swept disturbance gradient. Oecologia 105:484-492.

Matthiessen, B., and H. Hillebrand. 2006. Dispersal frequency affects local biomass production by controlling local diversity. Ecology Letters 9:652-662.

McGrady-Steed, J., P. M. Harris, and P. J. Morin. 1997. Biodiversity regulates ecosystem predictability. Nature 390:162-165.

Mouquet, N., and M. Loreau. 2002. Coexistence in metacommunities: the regional similarity hypothesis. American Naturalist 159:420-426.

Mouquet, N., and M. Loreau. 2003. Community patterns in source-sink metacommunities. American Naturalist 162:544557.

Muneepeerakul, R., J. S. Weitz, S. A. Levin, A. Rinaldo, and I. Rodriguez-Iturbe. 2007. A neutral metapopulation model of biodiversity in river networks. Journal of Theoretical Biology 245:351-363.

Namba, T., and C. Hashimoto. 2004. Dispersal-mediated coexistence of competing predators. Theoretical Population Biology 66:53-70.

Nathan, R., W. M. Getz, E. Revilla, M. Holyoak, R. Kadmon, D. Saltz, and P. E. Smouse. 2008. A movement ecology paradigm for unifying organismal movement research. Proceedings of the National Academy of Sciences USA 105:19052-19059.

Ovaskainen, O., and I. Hanski. 2002. Transient dynamics in metapopulation response to perturbation. Theoretical Population Biology 61:285-295.

Peterson, G. D. 2002. Contagious disturbance, ecological memory, and the emergence of landscape pattern. Ecosystems 5:329-338.

R Development Core Team. 2008. R: a language and environment for statistical computing. Version 2.8.0. R Foundation for Statistical Computing, Vienna, Austria. 〈www.r-project.org

Ricklefs, R. E. 2008. Disintegration of the ecological community. American Naturalist 172:741-750.

Salomon, Y., S. R. Connolly, and L. Bode. 2010. Effects of asymmetric dispersal on the coexistence of competing species. Ecology Letters 13:432-441.

Shea, K., S. H. Roxburgh, and E. S. J. Rauschert. 2004. Moving from pattern to process: coexistence mechanisms under intermediate disturbance regimes. Ecology Letters 7:491-508.

Sousa, W. P. 1984. The role of disturbances in natural communities. Annual Review of Ecology and Systematics 15:353-392.

Starzomski, B. M., and D. S. Srivastava. 2007. Landscape geometry determines community response to disturbance. Oikos 116:690-699.

Urban, M. C. 2004. Disturbance heterogeneity determines freshwater metacommunity structure. Ecology 85:2971-2978.

Vuilleumier, S., and H. P. Possingham. 2006. Does colonization asymmetry matter in metapopulations? Proceedings of the Royal Society B 273:1637-1642.

Warren, P. H. 1996. Dispersal and destruction in a multiple habitat system: an experimental approach using protist communities. Oikos 77:317-325.

\section{APPENDIX}

Figures showing results of additional simulations done with different initial parameter values, showing species traits for the main simulations and showing the percentage of occupied patches within the experimental protozoan metacommunities (Ecological Archives E092-074-A1). 